\title{
Menstrually Related Disorders: Points of Consensus, Debate, and Disagreement
}

Uriel Halbreich, M.D., John Bancroft, M.D., Lorrain Dennerstein, M.D., Jean Endicott, Ph.D., Fabio Faccinetti, Ph.D., Andrea Genazzani, M.D., Carol Morse, Ph.D., Barbara Parry, M.D., David Rubinow, M.D., Robert Reid, M.D., Isaac Schiff, M.D., Samuel Smith, M.D., and Torbjorn Bäckstrom, M.D.

Menstrually Related Disorders (MRDs) are quite prevalent, and in some women they are severe enough to warrant treatment. In most of the literature and in clinical practice, the focus has been on the premenstrual period, and most frequently the descriptive diagnosis given reflects that focus (e.g., Premenstrual syndrome [PMS]). Advances in the understanding of MRDs and formulation of adequate treatment when warranted, are hindered by the lack of agreed upon definitions and diagnostic criteria. The etiology and pathophysiology of the various MRDs are still unknown, and there are no specific medications or somatic treatments for sufferers that have been approved by the American Food and Drug Administration or any other major regulatory agency.

A constructive step toward progress in the understanding and eventual treatment of MRDs can be taken by the delineation of points of consensus and controversy. A discussion group on MRDs was held at the annual meeting of the American College of Neuropsychopharmacology in December 1991. Written opinions were presented by investigators from five countries: five

From the State University of New York (UH), Buffalo, New York; Medical Research Center Council (JB), Edinburgh, United Kingdom; University of Melboume (LD, CM), Australia; Columbia University (E), New York; University of Modena (FF), Italy; University of California (BP), San Diego, California; National Institute of Mental Health (DR), Bethesda, Maryland; University of Kensington (RR), Canada; Harvard University (IS), Boston, Massachusetts; Sinai Hospital of Baltimore (SS), Maryland; and Uppsala University (TB), Sweden.

Address correspondence to: Uriel Halbreich, M.D., State University of New York at Buffalo, 462 Grider Street, SUNY Clinical Center, Buffalo, New York 14215.

Received May 11, 1992; revised December 4, 1992; accepted January 27, 1993. psychiatrists, four gynecologists, two psychologists, and a biochemist (an additional gynecologist added his written opinion following the meeting). All have been active and published investigators in the field.

The report has been sent to several active distinguished investigators in the field who were not present in the group discussion and were anticipated to have different opinions on at least some of the points. We specifically tried to get responses from those who would not agree to the concept of MRDs. Rather, we hoped that they will make the point for emphasizing the premenstrual period and PMS. We also tried to get differing opinions on pathophysiology and treatment. Four people responded. Their written opinions follow the report of the discussion group. A general response to these opinions, written by the organizer of the discussion group (Uriel Halbreich) concludes the group of statements.

The following is a summary of the points on which the group felt that there was a consensus as well as the points on which a debate evolved and the only agreement was to disagree. We did not attempt to produce any specific proposal. The existence of symptoms and signs whose appearance or changes in severity are menstrually related was not questioned. The possible political ramifications of considering situations to be "disorders" were not the subject of the scientific discussion.

\section{THE NAME}

\section{Consensus}

Menstrually Related Disorder(s) (MRDs) has been agreed upon as a general term that applies to the vari- 
ety of conditions whose timing appears to be related to the menstrual cycle. One participant felt that "Menstrual Cycle-Related Disorder" might be a more adequate term. Another was bothered by the possible implication that MRD might be perceived as indicating a direct menstrual-bleeding effect rather than just timing of occurrence.

Most of the group were dissatisfied with the use of "Premenstrual Syndrome" (PMS) or its derivatives, as well the use of "Late Luteal Phase Dysphoric Disorder" (LLPDD), because symptoms are not limited to the premenstrual or late luteal phase. They can be perimenstrual, or they might persist for most of the luteal phase or may appear during the periovulatory period.

\section{DIAGNOSIS AND DIAGNOSTIC CRITERIA}

\section{Consensus}

The key elements of the conditions in focus are 1) cyclicity and recurrence; 2) temporal and consistent link to any phase of the menstrual cycle, mostly involving the perimenstrual and the periovulatory periods; 3 ) a need for prospective confirmation of initial complaints and their cyclical associations with the menstrual cycle; 4 ) to beconsidered a disorder, the condition(s) should also be characterized by clinically significant distress and a demonstration of associated impairment in functioning; 5) symptoms are diversified, involving many body systems; and 6) main symptoms are mostly consistent within each individual woman, although their severity may fluctuate across cycles.

\section{Points of Disagreement}

1) Should a specific MRD (or some subtypes of MRDs) be classified as a mental disorder?

2) Should an overall diagnosis be developed for all MRDs across the various body systems? If yes, under which classification umbrella? Currently, the ICD 9 and 10 include an $\mathrm{Ob} / \mathrm{Gyn}$ category for the gynecological condition, PMS (with no detailed definitions). There is no generalized category of diagnoses across specialties, for example, a diagnostic umbrella for menstrually related mood disorders, catamenial seizures, menstrual migraines, and premenstrual exacerbations of asthma or herpes.

Should an MRD be defined as a symptom or a syndrome (e.g., depression, migraine) secondary to, or associated with, a physical state (that would be the main diagnostic determinant), or should it be defined as "menstrually related" (dysphoria, migraine, etc.) as a main determinant of diagnosis?

\section{ETIOLOGY AND PATHOPHYSIOLOGY}

\section{Consensus}

There was agreement that 1) the pathophysiology of MRDs is likely to be multidimensional and multifactorial, involving various physiologic and biochemical systems; 2 ) there is a high likelihood of an involvement of the hypothalamo-pituitary-gonadal (HPG) system, although the nature of that involvement is not yet clear; 3 ) vulnerability of affected patients plays a major role in development of specific subtypes and symptoms; and 4) environmental and psychologic factors probably contribute to the development of symptoms as well as determination of their severity. The mechanism of this interaction is unknown.

\section{Points of Debate}

1) What is the actual role of gonadal hormones and other components of the HPG system in symptom formation of MRDs? Is there any significance to absolute levels of hormones, cyclicity, or rate of fluctuation? Is there hypersensitivity of receptors to gonadal hormones? Are there deviate metabolites in affected women?

2) What is the role of ovulation in the pathophysiology of MRDs?

3) Is there a monthly oscillator in women, independent of the regulatory mechanism of the HPG system?

4) What is the link between the pathophysiology of MRDs and changes in activity of neurotransmitters that putatively are significantly involved in the regulation of mood and behavior?

5) Is there a cyclic change of threshold for surfacing of symptoms? If yes, how is it associated with factors determining the vulnerability to develop symptoms?

6) What is the trigger of the pathophysiologic process(es) leading to the development of symptoms?

\section{TREATMENT OF MRDs}

\section{Consensus}

There is agreement that 1) ideally, treatment should be based on known etiology and pathophysiology, and that this ideal state has not been achieved yet for MRDs; 2) currently, two effective types of treatment modalities of MRDs have emerged - pharmacologic elimination of ovulation, which is probably effective as treatment for a wide variety of MRDs, and symptomatic treatments (e.g., several antidepressants, anxiolytics, antiepilepsy medications), which have been shown to be effective for specific target symptoms.

Some agreement was reached on the fact that a mu- 
tidisciplinary evaluation and treatment is needed for any patient suspected of having an MRD.

\section{Points of Debate}

1) What is the role and efficacy of psychosocial interventions for some types of MRDs?

2) What is the recommended "decision tree" and sequence of treatment options? Should the treatment choice and sequence of options depend upon the primary presenting complaints? Do we have sufficient data for any informed choice of treatment?

\section{SUMMARY}

It seems that during the past decade we have been witnessing an evolution of a consensus on the phenomenology and time course of various types of MRDs. We are in a stage in which definitions and diagnostic criteria can be developed, but their broad acceptance is still not assured. The etiology and pathophysiology are still fiercely debated, but reasonable and feasible methods for scientific elucidation of the various hypotheses are in place and are followed by solid groups. Despite the uncertainty concerning the etiology of MRDs, reasonably efficient treatment modalities do exist, and most sufferers of MRDs should expect an eventual alleviation of their symptoms if they are treated in a specialized, established, and up-to-date program. 